\title{
Le interviste INN-possibili: Ascolto prima di comunicare
}

\begin{abstract}
Bruno Bertone è nato professionalmente come trainer dell'area farmaceutica e per tale motivo ha tenuto corsi presso molti ospedali del nostro Paese, per numerosi gruppi di medici di medicina generale e per specialisti di varie branche, piuttosto che per associazioni mediche e di infermieri. Per meriti sul campo ha quindi conseguito un incarico presso l'Università degli Studi di Parma, Facoltà di Farmacia, ove è stato professore a contratto per dieci anni insegnando Comunicazione.

Ho conosciuto Bruno solo di recente in occasione di un Corso sulla Comunicazione agli infermieri di Nefrologia, Dialisi e Trapianto ove, dopo averlo introdotto all'argomento in nefrologia, l'ho seguito come discente. Grazie a lui sono tornato a capire l'importanza fondamentale che ha la comunicazione, in generale e in particolare nell'assistenza al paziente.

Vista la mia recidività e l'interesse che tale evento ha suscitato ho deciso di intervistarlo per la rubrica "Le Interviste INN-possibili”. L'approfondimento della sua conoscenza e una presa di coscienza ancora maggiore della frequenza con cui certi comportamenti latitano, mi hanno indotto a chiedergli di collaborare per il 2011 con il Giornale di $T N \& D$. Bruno terrà una rubrica in cui tratterà l'argomento applicato alla nostra realtà ma si è reso anche disponibile ad interagire con i componenti dell'area di Nursing nefrologico e con eventuali pazienti primi tra tutti quelli afferenti ad AIRP, per rispondere alle loro domande in modo da dare un risvolto di massima praticità alla rubrica, che abbiamo deciso di denominare: Ascolto prima di comunicare.

Buona lettura a tutti.
\end{abstract}

Marco Lombardi

\section{Intervista a Bruno Bertone}

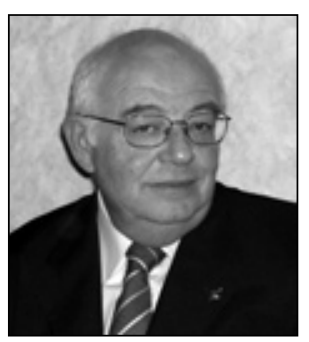

Dr. Bruno Bertone

bruno_bertone@virgilio.it

Tu ti occupi di comunicazione col paziente, sei medico?

In effetti non sono medico, ho conseguito una laurea in scienze biologiche nel lontano 1973, ma è la mia storia lavorativa che mi ha portato ad occuparmi dell'argomento.

\section{Quindi il tuo non è un interesse diretto?}

Se per "interesse diretto" intendi che non ho il problema di comunicare col paziente è così. In effetti mi sono interessato prima di comunicazione in termini generali e solo in seguito di comunicazione/relazione col paziente.
Ma non mi hai detto da dove derivi questo tuo interesse.

Ho fatto per anni il trainer (addestratore) in un'azienda farmaceutica e da esigenze professionali è nato un interesse personale per la comunicazione in generale. In un secondo tempo, dovendo costruire i messaggi per la promozione di farmaci per il trattamento di malattie croniche, è nato anche l'interesse per la comunicazione medico-paziente. $\grave{E}$ opportuno dire che molte malattie croniche richiederebbero quella che viene definita " $e d u$ cazione del paziente", una vera e propria formazione del paziente alla malattia che lo affligge. Lo scopo dell'“educazione del paziente" è fare in modo che questi riesca a convivere con la propria malattia e sia in grado di evitare quelle complicazioni che possono dipendere da un suo comportamento, con particolare riguardo allo stile di vita e all'adesione alla terapia. Una corretta comunicazione col paziente è un presupposto indispensabile a un'efficace "educazione".

Poi ti chiederò ancora della comunicazione medico-paziente, ma ora chiariscimi: com'è possibile che una perso- 
na laureata in una materia scientifica (biologia) arrivi a interessarsi di comunicazione.

Ti rispondo ben volentieri. Quando mi iscrissi all'università era mia intenzione fare il biologo, conseguita la laurea capii che, volendo lavorare, potevo fare l'informatore scientifico, il laboratorista o l'insegnante, sicuramente non il biologo: scelsi la prima opzione e per dieci anni feci l'informatore scientifico. Quando riuscii a realizzare il maggior fatturato e il maggior incremento di fatturato della rete mi resi conto che avevo raggiunto un traguardo, mi servivano nuove motivazioni. Mi candidai a una posizione di trainer presso la sede dell'azienda. Ben presto accanto al ruolo di trainer interno (addestramento di informatori) cominciai, sulle orme del mio responsabile, a tenere corsi sulle infezioni ospedaliere in molti ospedali della Penisola. Era il 1988 e tutti, se si proponeva loro un corso gratuito, erano ben felici di accettare. Sorse un problema, riempivamo le aule di infermieri mentre pochi, anzi pochissimi erano i medici che accettavano che un farmacista (il mio capo) e un biologo parlassero loro di infezioni. Noi volevamo avere in aula dei medici perché sarebbe stato un modo per promuovere il marchio aziendale.

Per attrarre medici in aula avremmo prima dovuto acquistare credibilità noi come persone, al di là dei nostri titoli di studio. Era necessario un corso che trattasse un tema lontano dagli interessi professionali del medico: "diagnosi e terapia”. Il modo per acquisire credibilità presso i medici fu un corso sulla comunicazione: "Comunicare in Pubblico". Chi seguiva quel corso era poi, di norma, disposto a seguirne anche uno sulle infezioni ospedaliere, questo perché chi lo teneva era considerato "credibile".

$\mathrm{E}$ in questo modo che ho approfondito i temi della comunicazione e ho tenuto decine di corsi "Comunicare in Pubblico" (e più di 100 i corsi sulle infezioni ospedaliere).

Nel 1995 fui chiamato dall'università di Parma, facoltà di Farmacia, a tenere un corso di "Metodologia della comunicazione" agli studenti del corso di laurea per informatori scientifici. Successivamente il corso venne esteso a farmacisti ed erboristi. Ho lasciato nel 2005, quindi per dieci anni ho potuto insegnare un argomento che mi appassionava e coinvolgeva.

\section{Ora mi dici della comunicazione medico-paziente?}

Linteresse per la comunicazione medico paziente nacque all'inizio degli anni Novanta. In azienda si sarebbe dovuto presentare alla classe medica un preparato per una malattia cronica (l'asma) nel giro di un paio di anni e io iniziai a interessarmi della comunicazione di questa malattia. Scoprii che i pazienti asmatici dovrebbero "conoscere la loro malattia per gestirla al meglio" ("edu- cazione del paziente"). Scoprii anche che gli asmatici, in generale, hanno tendenza a trattare le fasi di riacutizzazione della malattia, in pratica trattano il sintomo, tendono invece a non seguire quella che viene detta "terapia di fondo" che è cronica ed è intesa contenere la progressione di malattia (difficile assumere farmaci in assenza di sintomi!). In effetti se i pazienti con malattie croniche seguissero correttamente le terapie loro prescritte aumenterebbero di un buon $20 / 30 \%$, almeno, i fatturati dei farmaci indicati per quelle patologie. L'interesse iniziale era dovuto principalmente a motivi commerciali. Resta il fatto che se i pazienti affetti da malattie croniche fossero "educati" ed aderissero alle terapie potrebbero avere una miglior qualità di vita e al contempo farebbero aumentare i volumi di vendita di numerosi farmaci.

Dove e da chi hai appreso sulla comunicazione medicopaziente e sull'educazione del paziente?

Il primo argomento che ho affrontato nei primi anni Novanta è stato quello dell'educazione del paziente. Volevo capire se si potesse usare quel tema per fare consulenza ai medici. Per capire qualcosa di educazione del paziente ho seguito tre corsi della fondazione Smith Kline tenuti da Jean François d'Invernois, consulente dell'OMS per l'educazione del paziente e numero uno in Europa e nei paesi francofoni d'Africa. D'Invernois si è occupato in modo particolare di educazione dei pazienti diabetici. In seguito ho avuto modo di conoscere il Professor Aldo Moja (Università di Milano, cattedra di Psicologia medica presso l'Ospedale S. Paolo) che mi ha aperto il mondo della comunicazione medicopaziente. Le mie conoscenze su comunicazione, relazione ed educazione del paziente sono lungi dall'essere complete ma mi consentono di fare almeno opera di sensibilizzazione al tema presso medici e infermieri.

Se "comunicazione col paziente ed educazione del paziente" fossero tanto importanti ci sarebbe un corso per la facoltà di Medicina, non ti pare?

È dall'inizio degli anni'90 che il corso di "Psicologia medica" è obbligatorio nel corso di laurea in Medicina di tutte le facoltà. Nel corso gli studenti dovrebbero apprendere abilità di comunicazione e relazione con i pazienti. Ciò non di meno pochi sono i corsi di "Psicologia medica" che si occupano realmente di comunicazione relazione "medico-paziente" e danno allo studente le basi per condurre un efficace dialogo col paziente. Sarebbe molto importante che si formassero meglio gli studenti di medicina alla comunicazione/relazione col paziente. Un medico in grado di relazionarsi e comunicare correttamente soddisfa maggiormente le esigenze del paziente e professionalmente è più appagato. 